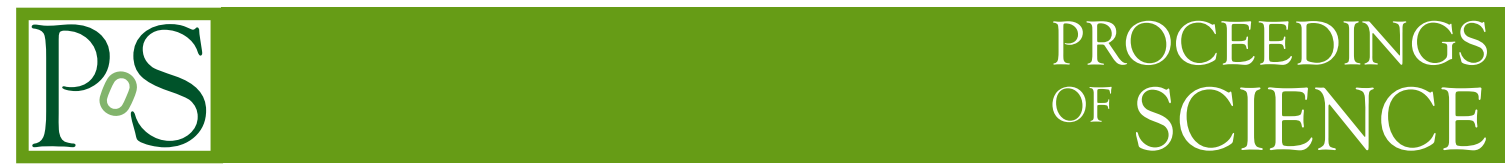

\title{
Groups of galaxies
}

\author{
Angela lovino ${ }^{* \dagger}$ \\ Osserv. Astron. di Brera - INAF Italy \\ E-mail: iovino@brera.mi.astro.it
}

\begin{abstract}
A large fraction of all galaxies in the universe are found in groups. They are the basic building blocks of the non-clustered part of the large scale structure, bridging intermediate scales between isolated galaxies and rich clusters. The study of galaxy groups is also important for investigating the relevant processes affecting galaxy formation and, particularly, evolution. Despite the importance and numbers of groups they are still much less studied than clusters of galaxies, as identification of structures with small numbers of galaxies is a difficult task. The recent availability of large databases (both photometric only and with redshift) is the key to exploiting the potentials of groups as cosmological tool. I will review some of the recent progress on this topic.
\end{abstract}

Baryons in Dark Matter Halos

5-9 October 2004

Novigrad, Croatia

${ }^{*}$ Speaker.

$\dagger$ 


\section{Introduction}

Groups of galaxies are interesting for a variety of reasons: they are tracers of the large scale structure of the universe, they provide sites in which to study the environmental dependence of galaxy properties, the small scale clustering of galaxies and the interactions between galaxies and hot/cold intragroup gas. It is therefore highly desirable to have extensive, homogeneous catalogs of groups of galaxies representing the variegated hierarchy of bound systems in the local/distant universe. The majority of galaxies resides in groups rather than clusters, that are a more elitist environment. Unfortunately groups are more difficult to detect than clusters, representing a smaller overdensity with respect to the background galaxy population. Today large redshift surveys (2dF, SLOAN and the ongoing VIMOS and DEEP2) allow us for the first time to select large numbers of galaxy aggregates covering a broad range in density enhancements and velocity dispersions, and to fully exploit the scientific potentials of groups. In parallel, a wealth of studies has expanded to the $\mathrm{X}$ rays and the radio the window of observations for groups, with detailed studies of smaller samples. This paper is divided in three main sections: I will start by presenting an overview of the new results that are coming from the $2 \mathrm{dF}$ and SLOAN group catalogs, where the statistical richness of the sample is the big advantage. I will then move to the results that are available on smaller samples of poor groups. Finally I will briefly present some promising new catalogs of groups.

\section{Galaxy groups from large redshift surveys}

The 2dF Galaxy Redshift Survey (GRS) and the SLOAN redshift survey are the result of a massive effort to acquire redshifts for a magnitude limited sample of galaxies, down to $\mathrm{b}_{j} \sim 19.45$ and $r \sim 17.77$ for $2 \mathrm{dF}$ and SLOAN respectively (see [1] and [2]). Both galaxy catalogs cover large areas on the sky (1300 sq. deg for $2 \mathrm{dF}$ and $4000 \mathrm{sq}$ deg for SLOAN) and have proved an unvaluable mining material to search for galaxy groups. Three different teams have already published group catalogs obtained from applying different finding algorithms to the 2dFGRS catalog (a paper on the algorithm used to detect groups from the SLOAN redshift catalog is in preparation).

A first selection was performed by [3] on the first 100k public data release of the $2 \mathrm{dF}$ data, by applying a modified Huchra and Geller group finding algorithm, while [4] applied a friend of friends percolation algorithm to the final release of the 2dFGRS. Both teams fine tuned their algorithm parameters by using cosmological simulations to produce mock versions of the 2dFGRS data. These simulations were used to check the reliability of the results obtained, and to pin down the best choice of parameters in order to grant unbiased estimates of velocity dispersion and sizes of the groups detected with respect to those of the undelying distribution of matter. [5] applied a different algorithm to the final 2dFGRS catalog: an halo-based group finder, which assigns galaxies to groups by iteration according to their common halos. The idea is to assume a mass-to-light ratio to assign a tentative mass to each group candidate, and then use this mass to re-determine group membership based on the newly estimated group size and velocity dispersion.

We will not enter into the details of the relative goodness of the different algorithms used, we will just mention that while the algorithm used by [4] and [3] is supposed to be more complete at the cost of a higher contamination by spurious groups, the algorithm adopted by [5] is less 
prone to contaminants, but possibly less complete (and this results in a slightly different number of groups extracted using the two strategies). Despite the different methods used, though, the general properties of the groups selected are similar: same median redshift $z \sim 0.11$ and median velocity dispersion $\sigma \sim 260 \mathrm{~km} \mathrm{sec}^{-1}$ for groups with more than 4 members, for a grand total of more than 2500 groups; for statistical studies these three large group catalogs are excellent. An interesting number: more than 55\% of galaxies are found in bound structures with 2 or more members, and this result holds irrespective of the algorithm used.

\subsection{Clustering of galaxy groups}

Groups play an important role in the hyerarchical large scale distribution of matter as they are simpler tracer of dark matter halo distribution than galaxies, and therefore easier to compare with model predictions. While the number of galaxies in each dark matter halo depends strongly on halo mass, every halo above some mass should correspond to a unique galaxy group.

From the observational point of view, as usual, things are more complicated: the small number of galaxies involved in individual groups makes it hard to uniquely identify systems that are physically associated, bound. Despite these problems the results obtained by different teams on the clustering of galaxy groups are very similar and provide interesting insights. There is clear indication that the correlation length increases substantially with the luminosity of the sample of groups considered, a quantity that correlates well with the total mass of the group itself, ranging from 6 to $20 h^{-1} \mathrm{Mpc}$, while there is little change in the correlation function slope (see refs.[6], [7] and [8]). Plotting the correlation lenght, $r_{0}$, as a function of the mean inter-group separation of the different mass/luminosity classes, a nice agreement is found between the results obtained for galaxy groups and those obtained for clusters by [9]. Both groups and clusters are in the same hierarchy of distribution and this result can be compared with predictions of theories of galaxy formation and used to constrain them.

\subsection{Luminosity function of galaxy groups}

The measurement of the galaxy luminosity function in groups and its dependence on groups mass is another measurement of cosmological relevance. The results of 2dFGRS groups, see ref. [10], show that a Schechter function is a reasonable fit to the galaxy luminosity function of groups of different mass, and that the characteristic luminosity $L^{*}$ increases for more massive groups, while $\alpha$, the faint end slope, decreases slightly, implying that there is a smaller number of fainter galaxies in more massive clusters. The highest mass groups have the largest error bars in $L^{*}$ and $\alpha$ determination, due to their smaller number in group catalogs. Another fundamental measurement is that of $\Upsilon$, the mass-to-light ratio of groups, that in semi-analytical models reflects the efficiency of star formation in haloes of different masses. Prior to the 2dFGRS results, some measurements were already available in the literature (among others those of [11] and [12]) but usually on smaller samples, or on catalogs containing groups from a number of different surveys. The results based on the 2dFGRS groups catalog imply that at larger halo masses the efficiency of star formation decreases. The expected upturn at lower halo masses, around $M \sim 10^{12} M_{\odot}-$ the size of the local group - is not yet reached by their data. 


\subsection{The influence of group environment on galaxy population.}

The influence of environment on galaxy properties is a long debated problem, usually explored by comparing cluster and field environment. This issue has recently been analysed in detail in a series of papers using groups/cluster samples both from 2dFGRS and from SLOAN redshift data (see refs. [13] [14], [15]). The detection of a strong correlation between the distribution of the equivalend width of the $\mathrm{H}_{\alpha}$ line $-\mathrm{W}_{0}\left(\mathrm{H}_{\alpha}\right)$, a tracer of higher/lower Star Formation Rate - and local density is very clearly seen in the data, with a trend that holds irrespectively of the parameter chosen to characterize local density, and that is visible even when using a large scale (up to $5 \mathrm{Mpc}$ ) to characterize density (see [15]). There are two distinct galaxy populations: those with significant, on going, star formation, covering a broad range in $\mathrm{H}_{\alpha}$ strenghts, and those with little or no ongoing star formation, forming a conspicuous ridge around $\mathrm{W}_{0}\left(\mathrm{H}_{\alpha}\right) \sim 0$. The relative ratio of the two populations changes with environment (see fig. 3 in [15]). There are two interesting things to notice. 1 - There is a $\sim 30 \%$ of bright galaxies, without any sign of star formation even at the lowest densities outside the groups. 2- The distribution of $\mathrm{W}_{0}\left(\mathrm{H}_{\alpha}\right)$ for galaxies with strong equivalent width does not change significantly as a function of galaxy environment. There are star-forming galaxies in dense environments with normal star formation rates, unhinibited by their present day environment. To see a real depletion of strong $\mathrm{W}_{0}\left(\mathrm{H}_{\alpha}\right)$ galaxies one has to go to the center of rich clusters (see [14]).

The fact that the population of actively star forming galaxies seems to be weakly sensitive to the environment is a result that challenges theories in which it is the environment at the present day that induces transformations in galaxy properties. There could be various factors conspiring to produce such an apparent insensitivity (and it could be that other parameters, like morphology could be a better indicator of the effects that environment has on galaxies). But a possible, simple, explanation is that pre-processing of galaxies in galaxy pairs/groups is responsible for the trend observed today at $z \sim 0$. In this scenario the star formation properties of galaxy population are related only indirectly to their present environment.

\section{A possible evolutionary scenario}

It has been suggested in the literature that mergers or close tidal interactions of galaxies in smaller galaxy aggregates within the infall region of clusters could be responsible for modifying the SFR of galaxies in cluster outskirts. This interpretation could be extended to poorer structures than clusters. Interaction rates appear to increase going back in time (see eg. [16]). In a simple bottom-up scenario of structure evolution, at high redshifts small galaxy aggregates could start forming in high density regions. In these denser regions galaxy interactions take place and have a chance (and sufficient time) to modify galaxy properties. Such past pre-processing of galaxies could be responsible for galaxy properties as we see them today, when most of these galaxies have ended up in higher density environments.

In the framework of this simple scenario, the $30 \%$ of quiescent galaxies observed in the field could be explained as fossil groups, see [17], the remnants of merging of few nearby galaxies into a single massive object. In other words the supposed isolation that we observe today for these galaxies is a misleading representation of their environment. These galaxies could be the markers 
of locally very dense environments, where all bright, surrounding galaxies have merged into one - possibly the end product of some compact groups similar to those in Hickson catalog. A few observational facts seem to point in this direction. Their number density (see [17]) is roughly $1 / 4$ of the galaxies expected to be quiescent in the field, suggesting that the two classes of objects could at least partly overlap. Fossil groups have a higher X-ray luminosity than groups with respect to their optical luminosity and a strongly peaked X-ray emission has been observed in the case of the fossil group NGC 6482 (see CHANDRA observations in [18]), suggesting old ages for these systems.

In this simple scenario we should be observing in the local universe enhancement of galaxy activity in at least some of the groups: those where interactions/merging are still at work to originate the large scale trends discussed in the previous section. According to [15] there is marginal evidence that the highest velocity dispersion groups have a lower fraction of galaxies with $\mathrm{W}_{0}\left(\mathrm{H}_{\alpha}\right)$ $>4 \AA$ for their local density relative to lower velocity dispersion groups. A similar clue was also noticed by [19]: in their sample the brightest galaxies in low velocity dispersion groups $\left(\sigma<150 \mathrm{~km} \mathrm{sec}^{-1}\right)$ were marginally bluer in distribution than field galaxies of similar luminosity.

Both observations point to the same conclusion: in small velocity dispersion groups there is an excess of star forming galaxies, supporting the evidence that low velocity dispersion and high densities encourage interactions. Unfortunately neither SLOAN or 2dFGRS survey is able to probe adequately structures composed by a handful of bright galaxies with typical separations of the order of $100 \mathrm{Kpc}$ : at $z \sim 0.1$ the fiber positioning costraint ( $\sim 1^{\prime}$ for both surveys) corresponds to a linear distance of approximately $80 h^{-1} K p c$.

\section{Poor groups and compact groups of galaxies}

To investigate in detail the possible link between group environment and galaxy population, we need to zoom in on special subsets of groups of galaxies: those groups eligible for galaxygalaxy interactions. A natural choice is to go for groups with small intergalactic separations, a handful of bright $\left(M \sim M^{*}\right)$ galaxies, to check how they fit in the scenario schetched above. These groups will be immune from factors that are likely to be at work in clusters (ram pressure stripping or galaxy harrassment) and therefore can be used to probe directly how galaxy-galaxy low velocity encounters and merging are effective in shaping galaxy properties.

Compact groups of galaxies, as defined by Hikson in his pioneereing work [20] - small galaxy aggregates of high local density and well separated from nearby clusters, HCGs - seem to provide exactly the class of systems that we require. The drawbacks of Hickson catalog are well known (see [21]) and better samples, obtained with similar criteria on digitised plates galaxy catalogs, are available today (eg. [22], [23], [24]). Still the Hickson sample is the most extensively studied up to date. More than $75 \%$ of its groups show X-ray emission, see [25] and many signs of interactions are displayed by each bright member galaxy, see [26].

Selecting groups based on visual proximity on the sky of their brightest members and isolation appears to be an efficient way to get bound structures, but one should remember that compact groups defined this way are actually a composite class of objects. It has been shown by [27] and [28] that when going deeper in spectroscopy than the initial handful of bright galaxies pinpointed by the selection, different classes of compact groups emerge: those truly compact, those that show 
a cluster-like core + halo structure, and larger, loose, systems with no central concentration of galaxies. Even as far as X-ray properties are concerned, no clear distinction is possible between compact groups as defined by the Hickson sample and generic poor groups (see [29]).

In order to appropriately characterize the dynamical status of each system one needs to examine in detail the velocity field of the fainter population around the brighter members. It is useful to merge informations that come from waveleghts other than the optical ones, especially in those cases of truly isolated, poor, systems, where the relative absence of a numerous, faint, galaxy population makes it difficult to draw secure conclusions on system dynamics. Before obtaining this information one should avoid looking at compact groups as a special class of objects but just treat them simply as instances of poor groups.

\section{The influence of the environment of poor groups on galaxy population.}

A series of papers have studied the effect of the enfironment of poor groups on galaxy population, but studies of large statistical samples are still missing (but a project like GEMS looks promising).

The relationship between galaxy activity and group dynamics has been explored in [30] and [31] by Coziol and collaborators. The existence of a density-morphology-activity relation is confirmed both in 17 groups from the Hickson and in the new Southern Compact Groups (SCGs, ref. [22]) sample. The fraction of starburst galaxies drops when going from the group halo to the group core, and in the core of groups there is a raise in the fraction of AGN and LLAGN activity. Interestingly SCG cores contain more star forming galaxies and less non-emission galaxies than the core of Hickson groups: SCGs, selected in an automated way, could probe a wider range of physical properties than HCGs, that are biased towards denser, more easily detectable and probably more evolved groups.

Zabludoff and Mulchaey performed a redshift survey of the fainter galaxies surrounding 12 poor groups, 9 of which X-ray detected (see [32], [33] and [34]). Interestingly for the three non$\mathrm{X}$-ray groups no significant population was detected other than the brightest known members and their early type fraction is zero, while $\mathrm{X}$-ray groups show significant substructure, suggesting that groups, like clusters, could evolve hierarchically and that some are still accreting smaller structures today (see [36]).

In groups where the spectroscopic survey extends outside the small radius defined by the brightest member galaxies, the radial profile of velocity dispersion indicates that a significant fraction of the group mass lies outside the galaxies, in a common group halo, explaining why these groups have not yet merged in one central galaxy. The observed fraction of early type galaxies is proportional to the velocity dispersion of the group, similar to a relationship noted by Hickson in his sample - see [35]. This fraction in some groups is comparable with that observed in clusters, suggesting that as the group velocity dispersion increases (beyond $400 \mathrm{~km} \mathrm{sec}^{-1}$, going to clusters domain) mergers become rarer and therefore morphological transformation due to merging ceases.

A study of the 6 bright $\left(\log L_{x}>41.2\right) \mathrm{X}$-ray groups reveals the presence of morphologyradius relation: for each galaxy the bulge fraction decreases with increasing projected distance from the group center (see [37]) and the periphery of the groups is dominated by galaxies with significant OII emission. Contrary to expectation though, the disk dominated galaxies in groups 
are less asymmetric on average than their counterparts in the field and have a lower OII equivalent width. This observation is puzzling and suggests older ages for such groups and their member galaxies. [38] had already observed that X-ray selection could produce a sample of groups more dynamically evolved. In such groups a late type galaxy may have consumed more of its gas than a similar galaxy in the field, and interactions have probably occurred far in its past.

Indications that X-ray poor groups are indeed younger systems, still keeping memory of recent merging/interactions, are found in [39]. The X-ray faint groups luminosity function shows a dip, or depletion, of galaxies in the mag range $-19 \leq M_{B} \leq-17$, that can be reproduced in a simple scenario where few very bright galaxies seen at the bright end of the LF are due to merging of intermediate luminosity galaxies. In X-ray bright groups no dip is visible, possibly because these groups have gone through galaxy merging stage in the past and further infall has washed out memory of this effect.

All these considerations seem to suggest that in spiral dominated, low mass, groups - those that in the hierarchical scenario have formed more recently - one should observe a higher than the field star formation activity, a higher percentage of morphological disturbances and a spiral fraction that is as low or lower than the one typical of the field. Observations that seem to corroborate a possible evolutionary sequence from low velocity dispersion, spiral dominated, groups, where star forming activity is still present, to higher velocity dispersion, mostly early type, X-ray strong, groups is presented in [40]. A somewhat similar suggestion was put forward by [42] using radio data.

\section{Young galaxy groups: the missing link ?}

Out of the 17 Hickson compact groups studied by [27] only one group, HGC16, was found in an extremely active phase. At a distance of $3400 \mathrm{~km} \mathrm{sec}^{-1}$, with a velocity dispersion of $85 \mathrm{~km} \mathrm{sec}^{-1}$ (from 9 member galaxies) and an X-ray luminosity of $2.410^{40} \mathrm{~h}^{-2} \mathrm{erg} \mathrm{sec}^{-1}$, hosting one Seyfert galaxy, two LINERS and three starburst galaxies (see [41]), HCG16 can be the prototype of a group where galaxy pre-processing is taking place today.

More of such groups have been found in the SCGs sample, that probes a wider range of physical properties than the Hickson sample. A splendid example of an active group is SCG00184854. This group is formed by 4 spiral galaxies at a distance of $3400 \mathrm{~km} \mathrm{sec}^{-1}$, in a very tight configuration (radius of $1^{\prime} .5$ ). one showing a spectacular tidal tail (see figure 1). It is a truly isolated group: only a single galaxy with concordant velocity is found at distance of $10^{\prime}$ from the group center, and the velocity dispersion of the system is $67 \mathrm{~km} \mathrm{sec}^{-1}$ (from 5 members).

The equivalend widths of $\mathrm{H}_{\alpha}$ lines of member galaxies range from 40 to $100 \AA$, and there is a large number of HII regions in the tidal tail of the brightest galaxy (Temporin et al. in prep). This group, as far as I know, is the first instance of a spiral only group detected in the X-ray, from $\mathrm{XMM}$ data recently obtained there is evidence of presence of diffuse gas, at a luminosity of 1.2 $10^{40} \mathrm{~h}^{-2} \mathrm{erg} \mathrm{sec}^{-1}$ (Trinchieri et al. in prep). SCG0018-4854 is one of the lowest luminosity group detected until now, and it fits very well in the evolutionary picture schetched above.

Another similar case is SCG2315-4241 (also known as the Gru group), whose velocity dispersion is $38 \mathrm{~km} \mathrm{sec}^{-1}$. It shows no detectable diffuse $\mathrm{X}$-ray emission $\left(L_{x}<5.510^{40} \mathrm{~h}^{-2} \mathrm{erg} \mathrm{sec}^{-1}\right)$, but a complex HI structure with strong evidence of tidal distorsions, and evident activity in the member galaxies (see [43]). 

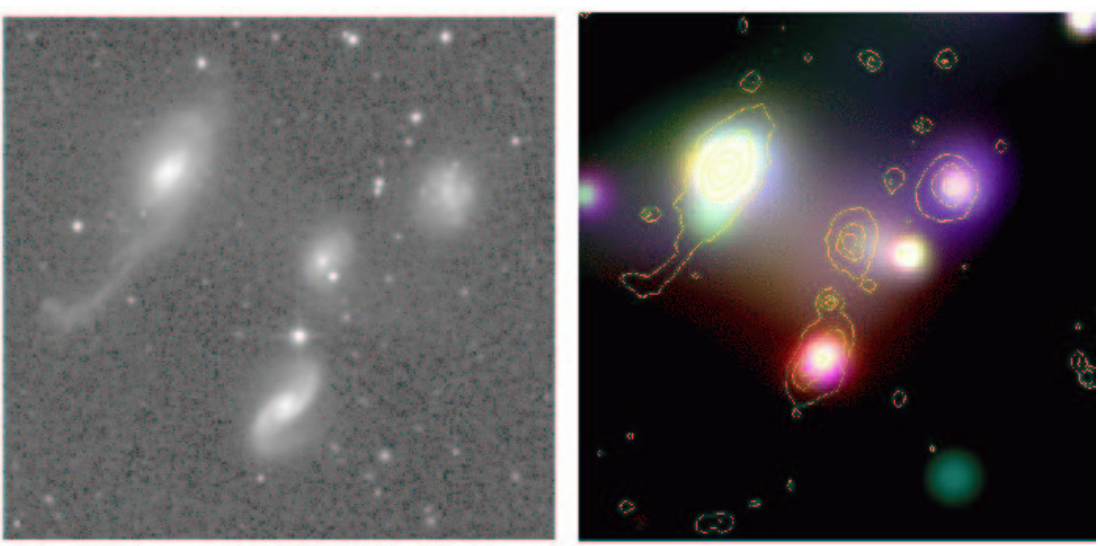

Figure 1: Group SCG0018-4854. Right panel:optical image. Left panel: XMM X-ray image with optical contours.

\section{Conclusions}

Searching for young groups in the local universe is a path to follow to get direct evidence for places where the environment is still shaping galaxy evolution. Another interesting possibility is to push to slightly higher redshifts (up to $z \sim 0.2$ ) the search for compact configurations on the sky. A new catalog has recently been obtained from the DPOSSII data (see [23], [44]) and a similar work has started in parallel on the SLOAN data (see [24]), aiming to expand at higher redshifts our knowledge of these objects. Spectroscopic follow-up is underway for the DPOSSII sample. In clusters we see evolution starting already at $z \sim 0.1-0.2$ : detecting evolutionary effects in these high density groups would provide further observational constraints for theories and models of the processes that drive galaxy evolution at low and intermediate redshifts.

\section{References}

[1] Colless, M. , et al., 2001, M.N.R.A.S., 328, 1039

[2] Strauss, M. A. , et al., 2002, A.J., 124, 1810

[3] Merchán, M. and Zandivarez, A., M.N.R.A.S., 2002, 335, 216

[4] Eke, V. R., et al., 2004, M.N.R.A.S., 348, 866

[5] Yang, X., et al., 2005, M.N.R.A.S., 356, 1293

[6] Zandivarez, A. and Merchán, M. E. and Padilla, N. D., 2003, M.N.R.A.S., 344, 247

[7] Padilla, N. D., et al., 2004, M.N.R.A.S., 352, 211

[8] Yang, X., et al., 2005, M.N.R.A.S., in press

[9] Bahcall, N. A., et al., 2003, Ap .J., 599, 814

[10] Eke, V. R., et al., 2004, M.N.R.A.S., 355, 769

[11] Marinoni, C. and Hudson, M. J., 2002, Ap .J., 569, 101

[12] Girardi, M., et al., 2002, Ap .J., 569, 720 
[13] Lewis, I. , et al., 2002, M.N.R.A.S., 334, 673

[14] Gómez, P. L., et al., 2003, Ap .J., 584, 210

[15] Balogh, M. L., et al., 2004, M.N.R.A.S., 348, 1355

[16] Conselice, C. J., et al., 2003, A.J., 126, 1183

[17] Jones, L. R., et al., 2003, M.N.R.A.S., 343, 627

[18] Khosroshahi, H. G. and Jones, L. R. and Ponman, T. J., 2004, M.N.R.A.S., 349, 1240

[19] Carlberg, R. G., et al., 2001, Ap.J., 563, 736

[20] Hickson, P., 1982, Ap .J., 255, 382

[21] Prandoni, I. and Iovino, A. and MacGillivray, H. T., 1994, A.J., 107, 1235

[22] Iovino, A., 2002, A.J., 124, 2471

[23] Iovino, A., et al., 2003, A.J., 125, 1660

[24] Lee, B. C., et al., 2004, A.J., 127, 1811

[25] Ponman, T. J., et al., 1996, M.N.R.A.S., 283, 690

[26] Mendes de Oliveira, C. and Hickson, P., 1994, Ap .J., 427, 684

[27] Ribeiro, A. L. B., et al., 1998, Ap .J., 497, 72

[28] de Carvalho, R. R., et al., 1997, Ap. J. Suppl., 110, 1

[29] Helsdon, S. F. and Ponman, T. J., 2000, M.N.R.A.S., 319, 933

[30] Coziol, R., et al., 1998, Ap.J., 506, 545

[31] Coziol, R. and Iovino, A. and de Carvalho, R. R., 2000, A.J., 120, 47

[32] Zabludoff, A. I. and Mulchaey, J. S., 1998, Ap .J., 496, 39

[33] Mulchaey, J. S. and Zabludoff, A. I., 1998, Ap .J., 496, 73

[34] Zabludoff, A. I. and Mulchaey, J. S., 2000, Ap .J., 539, 136

[35] Hickson, P. and Kindl, E. and Huchra, J. P., 1988, Ap .J., 331, 64

[36] , Zabludoff, A. I. and Mulchaey, J. S., 1998, Ap. J. Lett., 498, L5

[37] Tran, K. H., et al., 2001, Ap .J., 549, 172

[38] Henry, J. P., et al., 1995, Ap .J., 449, 422

[39] Miles, T. A., et al., 2004, M.N.R.A.S., 355, 785

[40] Coziol, R. and Brinks, E. and Bravo-Alfaro, H., 2004, A.J., 128, 68

[41] Ribeiro, A. L. B., et al., 1996, Ap. J. Lett., 463, L5

[42] Verdes-Montenegro, L., et al., 2001, A. \& A., 377, 812

[43] Dahlem, M., 2005, A. \& A., in press.

[44] de Carvalho, R. R., et al., 2005, A.J., submitted 